\title{
Weighted composition operators from weighted Bergman spaces with Békollé weights to Bloch-type spaces
}

\author{
Stevo Stević ${ }^{1,2^{*}}$ and Ajay K Sharma ${ }^{3}$
}

*Correspondence: sstevic@ptt.rs

${ }^{1}$ Mathematical Institute of the Serbian Academy of Sciences, Knez Mihailova 36/III, Beograd, 11000, Serbia

${ }^{2}$ Operator Theory and Applications Research Group, Department of Mathematics, King Abdulaziz University, P.O. Box 80203, Jeddah, 21589, Saudi Arabia

Full list of author information is available at the end of the article

\begin{abstract}
Let $\sigma$ be a Békollé weight function and $v$ be a weight function. In this paper, we characterize the boundedness and compactness of weighted composition operators acting from Bergman-type spaces $A^{\mathcal{P}}(\sigma)$ to Bloch-type spaces $\mathcal{B}_{v}$ and $\mathcal{B}_{v, 0}$ considerably extending some results in the literature.
\end{abstract}

MSC: Primary 47B33; 46E10; secondary 30D55

Keywords: weighted composition operator; Bergman-type spaces; Békollé weight; normal weight; Bloch-type spaces

\section{Introduction and preliminaries}

Let $\mathbb{D}$ denote the open unit disk in the complex plane $\mathbb{C}, H(\mathbb{D})$ the space of all holomorphic functions on $\mathbb{D}$, and $S(\mathbb{D})$ the class of all holomorphic self-maps of $\mathbb{D}$. Let $\psi \in H(\mathbb{D})$ and $\varphi \in S(\mathbb{D})$, the weighted composition operator $W_{\psi, \varphi}$ is a linear operator on $H(\mathbb{D})$ defined by

$$
W_{\psi, \varphi}(f)(z)=\psi(z) f(\varphi(z)), \quad z \in \mathbb{D}
$$

for $f \in H(\mathbb{D})$. It is of interest to provide function-theoretic characterizations when symbols $\varphi$ and $\psi$ induce a bounded or compact weighted composition operator between different function spaces. Recently, numerous authors have studied the boundedness and compactness of weighted composition operators on spaces of analytic functions on various domains (see, for example, [1-17] and the related references therein) as well as of some related operators involving composition ones on these spaces [18-26]. A joint feature for the majority of these papers is that they study the operators from or to Bloch-type or Bergman-type spaces. Paper [27] is one of the older papers that studied composition operators (case $\psi(z) \equiv 1$ ) on Bloch-type spaces and served as a motivation to several authors.

A continuous function $v: \mathbb{D} \rightarrow(0, \infty)$ is called weight. If $v(z)=v(|z|), z \in \mathbb{D}$, the weight is called radial. If a weight $v$ is such that $\lim _{|z| \rightarrow 1^{-}} v(z)=0$, we will call it a standard weight. Weight $v(r), r=|z|$, is called normal if there exist positive numbers $\eta$ and $\tau, 0<\eta<\tau$, and

(c) 2015 Stević and Sharma. This article is distributed under the terms of the Creative Commons Attribution 4.0 International License (http://creativecommons.org/licenses/by/4.0/), which permits unrestricted use, distribution, and reproduction in any medium, provided you give appropriate credit to the original author(s) and the source, provide a link to the Creative Commons license, and indicate if changes were made. 
$\delta \in[0,1)$ such that

$$
\begin{aligned}
& \frac{v(r)}{(1-r)^{\eta}} \text { is decreasing on }[\delta, 1) \text { and } \lim _{r \rightarrow 1} \frac{v(r)}{(1-r)^{\eta}}=0 ; \\
& \frac{v(r)}{(1-r)^{\tau}} \text { is increasing on }[\delta, 1) \text { and } \lim _{r \rightarrow 1^{-}} \frac{v(r)}{(1-r)^{\tau}}=+\infty .
\end{aligned}
$$

The classical weights $v_{\alpha}(r)=\left(1-r^{2}\right)^{\alpha}, \alpha>-1$, are obviously normal.

Let $d A(z)=d x d y / \pi=r d r d \theta / \pi$ stand for the normalized area measure in $\mathbb{D}$. As usual, a measurable function $g$ on $\mathbb{D}$ is called Lebesgue integrable if

$$
\int_{\mathbb{D}}|g(z)| d A(z)<\infty
$$

and it is written $g \in L^{1}(\mathbb{D})$. If $g \in L^{1}(\mathbb{D})$ is nonnegative, we will write $g \in L_{+}^{1}(\mathbb{D})$.

For $0<p<\infty$ and $\sigma$ a nonnegative Lebesgue integrable function on $\mathbb{D}$, we denote by $A^{p}(\sigma)$ the Bergman-type space consisting of all functions $f \in H(\mathbb{D})$ such that

$$
\|f\|_{A^{p}(\sigma)}^{p}=\int_{\mathbb{D}}|f(z)|^{p} \sigma(z) d A(z)<\infty .
$$

For $\sigma(z)=\left(1-|z|^{2}\right)^{\alpha}, \alpha>-1$, the space becomes the (standard) weighted Bergman space $A_{\alpha}^{p}(\mathbb{D})=A_{\alpha}^{p}$.

The weights considered here are the so-called Békollé weights [28], which are Bergman spaces analogues of the Muckenhoupt classes used in harmonic analysis. For $p_{0}>1$ and $\alpha>-1$, let $d A_{\alpha}(z)=(\alpha+1)\left(1-|z|^{2}\right)^{\alpha} d A(z), B_{p_{0}}(\alpha)$ be the class consisting of $\sigma \in L_{+}^{1}(\mathbb{D})$ with the property that there exists a constant $C>0$ such that

$$
\left(\int_{S(\theta, h)} \sigma(z) d A_{\alpha}(z)\right)\left(\int_{S(\theta, h)} \sigma^{-\frac{p_{0}^{\prime}}{p_{0}}}(z) d A_{\alpha}(z)\right)^{\frac{p_{0}}{p_{0}^{\prime}}} \leq C\left[A_{\alpha}(S(\theta, h))\right]^{p_{0}}
$$

for any Carleson square

$$
S(\theta, h)=\left\{z=r e^{i \phi}: 1-h<r<1,|\theta-\phi|<h / 2\right\}, \quad \theta \in[0,2 \pi], h \in(0,1),
$$

where $1 / p_{0}+1 / p_{0}^{\prime}=1$. It is not difficult to see that all normal weights are the Békollé weights.

It is well known [29] that the following inclusions hold:

$$
B_{p_{0}}\left(\alpha_{1}\right) \subset B_{p_{0}}\left(\alpha_{2}\right) \text { if }-1<\alpha_{1}<\alpha_{2}
$$

and

$$
B_{p_{0}}(\alpha) \subset B_{q_{0}}(\alpha) \quad \text { if } 1<p_{0}<q_{0}
$$

A function $\sigma \in L_{+}^{1}(\mathbb{D})$ belongs to class $C_{p_{0}}, p_{0}>1$, if there is a constant $C>0$ such that

$$
\left(\int_{D_{\lambda}(r)} \sigma(z) d A(z)\right)\left(\int_{D_{\lambda}(r)} \sigma^{-\frac{p_{0}^{\prime}}{p_{0}}}(z) d A(z)\right)^{\frac{p_{0}}{p_{0}^{\prime}}} \leq C\left[A\left(D_{\lambda}(r)\right)\right]^{p_{0}}
$$


for every disk $D_{\lambda}(r)=\{z \in \mathbb{D}:|z-\lambda|<r(1-|\lambda|)\}$. Here $r \in(0,1)$ is fixed, but the class $C_{p_{0}}$ is actually independent of $r \in(0,1)$. Moreover, $B_{p_{0}}(\alpha) \subset C_{p_{0}}$ for every $\alpha>-1$ and the inclusion is strict. For more about the classes $B_{p_{0}}(\alpha)$ and $C_{p_{0}}$ and the properties satisfied by the weights in these classes, we refer to [30] and [29] and the references therein.

Throughout this paper constants are denoted by $C$, they are positive and not necessarily the same at each occurrence. The notation $A \lesssim B$ means that $A \leq C B$ for some $C>0$ independent of the variables involved into these quantities. If $A \lesssim B$ and $B \lesssim A$, then we write $A \asymp B$.

For functions in $A^{p}(\sigma)$, when the weight $\sigma$ is in $C_{p_{0}}$, we have the following estimate, which easily follows by using a standard procedure, namely, from the Cauchy inequality applied to function $f^{(k)}$, the subharmonicity of $|f|^{p}, p>0$, the integral Hölder inequality and the definition of class $C_{p_{0}}$.

Lemma 1 Let $r \in(0,1), \sigma \in L_{+}^{1}(\mathbb{D}) \cap C_{p_{0}}, p_{0}>1, p>0$ and $k \in \mathbb{N}_{0}$. Then there is a constant $C>0$ independent of $z$ such that

$$
\left|f^{(k)}(z)\right| \leq C \frac{\left(\int_{D_{z}(r)} \sigma(\zeta) d A(\zeta)\right)^{-1 / p}}{\left(1-|z|^{2}\right)^{k}}\|f\|_{A^{p}(\sigma)}
$$

for every $f \in A^{p}(\sigma)$.

The next lemma provides an asymptotic estimate for the norm of weighted Bergman kernel (see [30]).

Lemma 2 Let $r \in(0,1)$ be fixed, $p>0, p_{0}>1$ and $\eta>-1$. Assume that $p_{0} \geq p, \sigma \in L_{+}^{1}(\mathbb{D})$ is such that $\sigma(z) /\left(1-|z|^{2}\right)^{\alpha}$ belongs to $B_{p_{0}}(\alpha)$ and $\gamma \geq(\eta+2) p_{0} / p-2$. Let

$$
K_{\lambda}^{\gamma}(z)=\frac{1}{(1-\bar{\lambda} z)^{\gamma+2}}, \quad \lambda \in \mathbb{D}
$$

(the reproducing kernel of the weighted Bergman space $A_{\gamma}^{p}$ ). Then

$$
\left\|K_{\lambda}^{\gamma}\right\|_{A^{p}(\sigma)} \asymp \frac{\left(\int_{D_{\lambda}(r)} \sigma d A\right)^{1 / p}}{\left(1-|\lambda|^{2}\right)^{\gamma+2}} .
$$

Lemma 3 Let $r \in(0,1)$ be fixed, $p>0, p_{0}>1$ and $\eta>-1$. Assume that $p_{0} \geq p, \sigma \in L_{+}^{1}(\mathbb{D})$ is such that $\sigma(z) /\left(1-|z|^{2}\right)^{\alpha}$ belongs to $B_{p_{0}}(\alpha)$. Then

$$
f_{\lambda}(z)=\frac{\left(1-|\lambda|^{2}\right)^{(\eta+2) p_{0} / p+1}}{\left(\int_{D_{\lambda}(r)} \sigma d A\right)^{1 / p}(1-\bar{\lambda} z)^{(\eta+2) p_{0} / p+1}}
$$

is in $A^{p}(\sigma)$. Moreover,

$$
\sup _{\lambda \in \mathbb{D}}\left\|f_{\lambda}\right\|_{A^{p}(\sigma)} \asymp 1,
$$

and $f_{\lambda}$ converges to zero uniformly on compact subsets of $\mathbb{D}$ as $|\lambda| \rightarrow 1^{-}$. 
Proof Let $\gamma=(\eta+2) p_{0} / p-2$. Then by Lemma 2 we have

$$
\left|f_{\lambda}(z)\right| \asymp \frac{\left|K_{\lambda}^{\gamma+1}(z)\right|}{\left\|K_{\lambda}^{\gamma+1}\right\|_{A^{p}(\sigma)}},
$$

from which (2) follows. By Lemma 1 with $k=0$ and Lemma 2, we have that

$$
1=\left|K_{\lambda}^{\gamma}(0)\right| \leq C\left(\int_{D_{0}(1 / 2)} \sigma d A\right)^{-1 / p}\left\|K_{\lambda}^{\gamma}\right\|_{A^{p}(\sigma)} \asymp \frac{\left(\int_{D_{\lambda}(r)} \sigma d A\right)^{1 / p}}{\left(1-|\lambda|^{2}\right)^{\gamma+2}} .
$$

Thus

$$
\frac{\left(1-|\lambda|^{2}\right)^{(\eta+2) p_{0} / p}}{\left(\int_{D_{\lambda}(r)} \sigma d A\right)^{1 / p}}=\frac{\left(1-|\lambda|^{2}\right)^{\gamma+2}}{\left(\int_{D_{\lambda}(r)} \sigma d A\right)^{1 / p}} \lesssim 1
$$

Using (3) in (1) it is easy to see that $f_{\lambda}$ converges to zero uniformly on compact subsets of $\mathbb{D}$, as $|\lambda| \rightarrow 1^{-}$.

For a weight $v$, the Bloch-type space $\mathcal{B}_{v}$ on $\mathbb{D}$ is the space of all holomorphic functions $f$ on $\mathbb{D}$ such that

$$
\sup _{z \in \mathbb{D}} v(z)\left|f^{\prime}(z)\right|<\infty
$$

The little Bloch-type space $\mathcal{B}_{v, 0}$ consists of all $f \in \mathcal{B}_{v}$ such that

$$
\lim _{|z| \rightarrow 1} v(z)\left|f^{\prime}(z)\right|=0
$$

Both spaces $\mathcal{B}_{v}$ and $\mathcal{B}_{v, 0}$ are Banach spaces with the norm

$$
\|f\|_{\mathcal{B}_{v}}=|f(0)|+\sup _{z \in \mathbb{D}} v(z)\left|f^{\prime}(z)\right|
$$

and $\mathcal{B}_{v, 0}$ is a closed subspace of $\mathcal{B}_{v}$

Depending on the weight $v$, various Bloch-type spaces are obtained. For $v(z)=\left(1-|z|^{2}\right)^{\alpha}$, $\alpha>0$, the spaces are reduced to the so-called $\alpha$-Bloch, that is, the little $\alpha$-Bloch space, which is for $\alpha=1$ reduced to the classical Bloch space. The reader could see that papers $[1-6,8,10-12,14,15,18-26]$ consider concrete operators on or to various Bloch-type spaces. Nowadays we know that if the image space is a Bloch-type, then usually it does not affect much on the boundedness and compactness, so we will here consider the spaces with as much as general weights.

The compactness of a closed subset $L \subset \mathcal{B}_{v, 0}$ can be characterized as follows.

Lemma 4 Let $v$ be a standard weight. A closed set $L$ in $\mathcal{B}_{v, 0}$ is compact if and only if it is bounded with respect to the norm $\|\cdot\|_{\mathcal{B}_{v}}$ and satisfies

$$
\lim _{|z| \rightarrow 1} \sup _{f \in L} v(z)\left|f^{\prime}(z)\right|=0
$$

This result for the case $v(z)=1-|z|^{2}$ was proved by Madigan and Matheson in [27]. By a slight modification of their proof in the case, Lemma 4 is proved. 
Motivated by [3], as well as by [9], here we characterize the boundedness and compactness of the weighted composition operators acting from the Bergman-type spaces $A^{p}(\sigma)$ to Bloch-type spaces $\mathcal{B}_{v}$ and $\mathcal{B}_{v, 0}$. Our results extend some in [3].

The following criterion for compactness follows by standard arguments which appeared for the first time in [31].

Lemma 5 Let $\sigma \in L_{+}^{1}(\mathbb{D})$ be such that $\sigma(z) /\left(1-|z|^{2}\right)^{\alpha}$ belongs to $B_{p_{0}}(\alpha)$, v be a standard weight and $W_{\psi, \varphi}: A^{p}(\sigma) \rightarrow \mathcal{B}_{\nu}$ be bounded. Then $W_{\psi, \varphi}: A^{p}(\sigma) \rightarrow \mathcal{B}_{\nu}$ is compact if and only if for any bounded sequence $\left(f_{n}\right)_{n \in \mathbb{N}}$ in $A^{p}(\sigma)$ which converges to zero uniformly on compact subsets of $\mathbb{D}$, we have

$$
\lim _{n \rightarrow \infty}\left\|W_{\psi, \varphi} f_{n}\right\|_{\mathcal{B}_{v}}=0
$$

2 Boundedness and compactness of $W_{\psi, \varphi}: A^{p}(\sigma) \rightarrow \mathcal{B}_{v}\left(\mathcal{B}_{v, 0}\right)$

In this section we formulate and prove the main results in this paper.

Theorem 1 Let $r \in(0,1)$ be fixed, $p>0, p_{0}>1, \alpha>-1$, v be a weight, $\psi \in H(\mathbb{D})$ and $\varphi \in$ $S(\mathbb{D})$. Assume that $p_{0} \geq p$ and $\sigma \in L_{+}^{1}(\mathbb{D})$ is such that $\sigma(z) /\left(1-|z|^{2}\right)^{\alpha}$ belongs to $B_{p_{0}}(\alpha)$.

Then $W_{\psi, \varphi}: A^{p}(\sigma) \rightarrow \mathcal{B}_{v}$ is bounded if and only if the following conditions are satisfied:

(i) $M_{1}=\sup _{z \in \mathbb{D}} v(z)\left|\psi^{\prime}(z)\right|\left(\int_{D_{\varphi(z)}(r)} \sigma d A\right)^{-1 / p}<\infty$;

(ii) $M_{2}=\sup _{z \in \mathbb{D}} \frac{v(z)\left|\psi(z) \varphi^{\prime}(z)\right|}{1-|\varphi(z)|^{2}}\left(\int_{D_{\varphi(z)}(r)} \sigma d A\right)^{-1 / p}<\infty$.

Moreover, if $W_{\psi, \varphi}: A^{p}(\sigma) \rightarrow \mathcal{B}_{v}$ is bounded, then

$$
\left\|W_{\psi, \varphi}\right\|_{A^{p}(\sigma) \rightarrow \mathcal{B}_{v} / \mathbb{C}} \asymp M_{1}+M_{2} .
$$

Proof First suppose that conditions (i) and (ii) hold. Then by Lemma 1 we have

$$
\begin{aligned}
v(z)\left|\left(W_{\psi, \varphi} f\right)^{\prime}(z)\right| \leq & v(z)\left|\psi^{\prime}(z)\right||f(\varphi(z))|+v(z)\left|\psi(z) \varphi^{\prime}(z)\right|\left|f^{\prime}(\varphi(z))\right| \\
\lesssim & \left(v(z)\left|\psi^{\prime}(z)\right|\left(\int_{D_{\varphi(z)}(r)} \sigma d A\right)^{-1 / p}\right. \\
& \left.+\frac{v(z)\left|\psi(z) \varphi^{\prime}(z)\right|}{1-|\varphi(z)|^{2}}\left(\int_{D_{\varphi(z)}(r)} \sigma d A\right)^{-1 / p}\right)\|f\|_{A^{p}(\sigma)} .
\end{aligned}
$$

Furthermore,

$$
\left|W_{\psi, \varphi}(f)(0)\right|=|\psi(0)||f(\varphi(0))| \lesssim|\psi(0)|\left(\int_{D_{\varphi(0)}(r)} \sigma d A\right)^{-1 / p}\|f\|_{A^{p}(\sigma)} .
$$

Using (i), (ii), (5) and (6), we see that

$$
\begin{aligned}
\left\|W_{\psi, \varphi} f\right\|_{\mathcal{B}_{v}} & =|\psi(0)||f(\varphi(0))|+\sup _{z \in \mathbb{D}} v(z)\left|\left(W_{\psi, \varphi} f\right)^{\prime}(z)\right| \\
& \lesssim\left(|\psi(0)|\left(\int_{D_{\varphi(0)}(r)} \sigma d A\right)^{-1 / p}+M_{1}+M_{2}\right)\|f\|_{A^{p}(\sigma)} .
\end{aligned}
$$

So, we have that $W_{\psi, \varphi}: A^{p}(\sigma) \rightarrow \mathcal{B}_{\nu}$ is bounded and

$$
\left\|W_{\psi, \varphi}\right\|_{A^{p}(\sigma) \rightarrow \mathcal{B}_{v} / \mathbb{C}} \lesssim M_{1}+M_{2} .
$$


Conversely, suppose that $W_{\psi, \varphi}: A^{p}(\sigma) \rightarrow \mathcal{B}_{v}$ is bounded. Then, by taking $f(z) \equiv 1 \epsilon$ $A^{p}(\sigma)$, we have that

$$
\sup _{z \in \mathbb{D}} v(z)\left|\psi^{\prime}(z)\right|=\left\|W_{\psi, \varphi}(1)\right\|_{\mathcal{B}_{v}} \lesssim\left\|W_{\psi, \varphi}\right\|_{A^{p}(\sigma) \rightarrow \mathcal{B}_{v}}
$$

By taking $f(z)=z \in A^{p}(\sigma)$, using the boundedness of $\varphi$ and asymptotic estimate (8), we easily get

$$
\sup _{z \in \mathbb{D}} v(z)\left|\psi(z) \varphi^{\prime}(z)\right| \lesssim\left\|W_{\psi, \varphi}\right\|_{A^{p}(\sigma) \rightarrow \mathcal{B}_{v}} .
$$

Let $\lambda=\varphi(\zeta), \zeta \in \mathbb{D}$, and $g_{\lambda}(z)=\tau_{\lambda}(z) f_{\lambda}(z)$, where $f_{\lambda}$ is defined in (1) and $\tau_{\lambda}$ is defined as

$$
\tau_{\lambda}(z)=1-\frac{1-|\lambda|^{2}}{1-\bar{\lambda} z}
$$

Then $\tau_{\lambda} \in H^{\infty}$ as

$$
\sup _{z \in \mathbb{D}}\left|\tau_{\lambda}(z)\right| \leq \sup _{z \in \mathbb{D}}\left(1+\frac{1-|\lambda|^{2}}{1-|\lambda||z|}\right) \leq 3 .
$$

Thus $g_{\lambda} \in A^{p}(\sigma)$ and $\sup _{z \in \mathbb{D}}\left\|g_{\lambda}\right\|_{A^{p}(\sigma)} \lesssim 1$. Moreover,

$$
\tau_{\lambda}(\lambda)=0, \quad \tau_{\lambda}^{\prime}(z)=-\bar{\lambda} \frac{1-|\lambda|^{2}}{(1-\bar{\lambda} z)^{2}} \quad \text { and } \quad \tau_{\lambda}^{\prime}(\lambda)=\frac{-\bar{\lambda}}{1-|\lambda|^{2}} .
$$

We also have

$$
\begin{aligned}
& f_{\lambda}(\lambda)=\left(\int_{D_{\lambda}(r)} \sigma d A\right)^{-1 / p} \\
& f_{\lambda}^{\prime}(z)=\bar{\lambda}\left((\eta+2) \frac{p_{0}}{p}+1\right) \frac{\left(1-|\lambda|^{2}\right)^{(\eta+2) p_{0} / p+1}}{\left(\int_{D_{\lambda}(r)} \sigma d A\right)^{1 / p}(1-\bar{\lambda} z)^{(\eta+2) p_{0} / p+2}}
\end{aligned}
$$

and

$$
f_{\lambda}^{\prime}(\lambda)=\bar{\lambda}\left((\eta+2) \frac{p_{0}}{p}+1\right) \frac{\left(\int_{D_{\lambda}(r)} \sigma d A\right)^{-1 / p}}{1-|\lambda|^{2}} .
$$

Therefore $g_{\lambda}(\lambda)=0$ and, from (11) and (12), we have

$$
g_{\lambda}^{\prime}(\lambda)=\tau_{\lambda}^{\prime}(\lambda) f_{\lambda}(\lambda)+\tau_{\lambda}(\lambda) f_{\lambda}^{\prime}(\lambda)=-\bar{\lambda} \frac{\left(\int_{D_{\lambda}(r)} \sigma d A\right)^{-1 / p}}{1-|\lambda|^{2}} .
$$

Using this fact we obtain

$$
\begin{aligned}
\left\|W_{\psi, \varphi}\right\|_{A^{p}(\sigma) \rightarrow \mathcal{B}_{v}} & \gtrsim\left\|W_{\psi, \varphi} g_{\varphi(\zeta)}\right\|_{\mathcal{B}_{v}} \\
& \geq v(\zeta)\left|\psi^{\prime}(\zeta) g_{\varphi(\zeta)}(\varphi(\zeta))+\psi(\zeta) \varphi^{\prime}(\zeta) g_{\varphi(\zeta)}^{\prime}(\varphi(\zeta))\right| \\
& \geq v(\zeta)\left|\psi(\zeta) \varphi^{\prime}(\zeta)\right||\varphi(\zeta)| \frac{\left(\int_{D_{\varphi(\zeta)}(r)} \sigma d A\right)^{-1 / p}}{1-|\varphi(\zeta)|^{2}}
\end{aligned}
$$


Thus, for a fixed $\delta \in(0,1)$, we obtain

$$
\sup _{|\varphi(\zeta)|>\delta} v(\zeta)\left|\psi(\zeta) \varphi^{\prime}(\zeta)\right| \frac{\left(\int_{D_{\varphi(\zeta)}(r)} \sigma d A\right)^{-1 / p}}{1-|\varphi(\zeta)|^{2}} \lesssim\left\|W_{\psi, \varphi}\right\|_{A^{p}(\sigma) \rightarrow \mathcal{B}_{\nu}}
$$

By using (3) and (9), we have that

$$
\begin{aligned}
& \sup _{|\varphi(\zeta)| \leq \delta} v(\zeta)\left|\psi(\zeta) \varphi^{\prime}(\zeta)\right| \frac{\left(\int_{D_{\varphi(\zeta)}(r)} \sigma d A\right)^{-1 / p}}{1-|\varphi(\zeta)|^{2}} \\
& \quad=\sup _{|\varphi(\zeta)| \leq \delta} \frac{v(\zeta)\left|\psi(\zeta) \varphi^{\prime}(\zeta)\right|}{\left(1-|\varphi(\zeta)|^{2}\right)^{(\eta+2) p_{0} / p+1}} \frac{\left(1-|\varphi(\zeta)|^{2}\right)^{(\eta+2) p_{0} / p}}{\left(\int_{\varphi(\zeta)(r)} \sigma d A\right)^{1 / p}} \\
& \quad \lesssim \frac{1}{\left(1-\delta^{2}\right)^{(\eta+2) p_{0} / p+1}}\left\|W_{\psi, \varphi}\right\|_{A^{p}(\sigma) \rightarrow \mathcal{B}_{v}} .
\end{aligned}
$$

Hence from (16) and (17) we have

$$
\sup _{\zeta \in \mathbb{D}} \nu(\zeta)\left|\psi(\zeta) \varphi^{\prime}(\zeta)\right| \frac{\left(\int_{D_{\varphi(\zeta)}(r)} \sigma d A\right)^{-1 / p}}{1-|\varphi(\zeta)|^{2}} \lesssim\left\|W_{\psi, \varphi}\right\|_{A^{p}(\sigma) \rightarrow \mathcal{B}_{\nu}} .
$$

Let $\lambda=\varphi(\zeta), \zeta \in \mathbb{D}$, and $f_{\lambda}$ be defined in (1). Recall that $\sup _{\lambda \in \mathbb{D}}\left\|f_{\lambda}\right\|_{A^{p}(\sigma)} \lesssim 1$. Hence, by (12) and (13) we get

$$
\begin{aligned}
& \left\|W_{\psi, \varphi}\right\|_{A^{p}(\sigma) \rightarrow \mathcal{B}_{v}} \gtrsim\left\|W_{\psi, \varphi} f_{\varphi(\zeta)}\right\|_{\mathcal{B}_{\nu}} \geq\left|\psi^{\prime}(\zeta) f_{\varphi(\zeta)}(\varphi(\zeta))+\psi(\zeta) \varphi^{\prime}(\zeta) f_{\varphi(\zeta)}^{\prime}(\varphi(\zeta))\right| \\
& \geq v(\zeta)\left|\psi^{\prime}(\zeta)\right|\left(\int_{D_{\varphi(\zeta)}(r)} \sigma d A\right)^{-1 / p} \\
& -\left((\eta+2) \frac{p_{0}}{p}+1\right) \frac{\nu(\zeta)|\psi(\zeta)|\left|\varphi^{\prime}(\zeta)\right|}{1-|\varphi(\zeta)|^{2}}|\varphi(\zeta)|\left(\int_{D_{\varphi(\zeta)}(r)} \sigma d A\right)^{-1 / p}
\end{aligned}
$$

from which, along with the boundedness of $\varphi$, it follows that

$$
\begin{aligned}
& \nu(\zeta)\left|\psi^{\prime}(\zeta)\right|\left(\int_{D_{\varphi(\zeta)}(r)} \sigma d A\right)^{-1 / p} \\
& \quad \leq\left\|W_{\psi, \varphi}\right\|_{A^{p}(\sigma) \rightarrow \mathcal{B}_{\nu}}+C \frac{\nu(\zeta)\left|\psi(\zeta) \| \varphi^{\prime}(\zeta)\right|}{1-|\varphi(\zeta)|^{2}}\left(\int_{D_{\varphi(\zeta)}(r)} \sigma d A\right)^{-1 / p}
\end{aligned}
$$

Taking the supremum over $\zeta \in \mathbb{D}$ in (19) and using (18), we get

$$
\sup _{\zeta \in \mathbb{D}} v(\zeta)\left|\psi^{\prime}(\zeta)\right|\left(\int_{D_{\varphi(\zeta)}(r)} \sigma d A\right)^{-1 / p} \lesssim\left\|W_{\psi, \varphi}\right\|_{A^{p}(\sigma) \rightarrow \mathcal{B}_{\nu}}
$$

From (18) and (20) we have

$$
M_{1}+M_{2} \lesssim\left\|W_{\psi, \varphi}\right\|_{A^{p}(\sigma) \rightarrow \mathcal{B}_{\nu} / \mathbb{C}}
$$

Finally, from (7) and (21), (4) holds. 
Theorem 2 Let $r \in(0,1)$ be fixed, $p>0, p_{0}>1, \alpha>-1$, v be a standard weight, $\psi \in H(\mathbb{D})$ and $\varphi \in S(\mathbb{D})$. Assume that $p_{0} \geq p, \sigma \in L_{+}^{1}(\mathbb{D})$ is such that $\sigma(z) /\left(1-|z|^{2}\right)^{\alpha}$ belongs to $B_{p_{0}}(\alpha)$ and $W_{\psi, \varphi}: A^{p}(\sigma) \rightarrow \mathcal{B}_{v}$ is bounded. Then $W_{\psi, \varphi}: A^{p}(\sigma) \rightarrow \mathcal{B}_{v}$ is compact if and only if the following conditions are satisfied:

(i) $\lim _{|\varphi(z)| \rightarrow 1} v(z)\left|\psi^{\prime}(z)\right|\left(\int_{D_{\varphi(z)}(r)} \sigma d A\right)^{-1 / p}=0$;

(ii) $\lim _{|\varphi(z)| \rightarrow 1} \frac{v(z)\left|\psi(z) \varphi^{\prime}(z)\right|}{1-|\varphi(z)|^{2}}\left(\int_{D_{\varphi(z)}(r)} \sigma d A\right)^{-1 / p}=0$.

Proof First suppose that conditions (i) and (ii) hold. Then by Lemma 5 it is sufficient to show that if $\left(f_{n}\right)_{n \in \mathbb{N}}$ is a bounded sequence in $A^{p}(\sigma)$ that converges to zero uniformly on compact subsets of $\mathbb{D}$, then $\left\|W_{\psi, \varphi} f_{n}\right\|_{\mathcal{B}_{v}} \rightarrow 0$ as $n \rightarrow \infty$. Let $\left(f_{n}\right)_{n \in \mathbb{N}} \subset A^{p}(\sigma)$ be such a sequence. By conditions (i) and (ii), we have that for any $\varepsilon>0$, there is $\delta \in(0,1)$ such that

$$
v(z)\left|\psi^{\prime}(z)\right|\left(\int_{D_{\varphi(z)}(r)} \sigma d A\right)^{-1 / p}<\varepsilon
$$

and

$$
\frac{v(z)\left|\psi(z) \varphi^{\prime}(z)\right|}{1-|\varphi(z)|^{2}}\left(\int_{D_{\varphi(z)}(r)} \sigma d A\right)^{-1 / p}<\varepsilon,
$$

whenever $\delta<|\varphi(z)|<1$.

Let $K=\{z \in \mathbb{D}:|z| \leq \delta\}$. Clearly, $K$ is a compact subset of $\mathbb{D}$. We have

$$
\begin{aligned}
\left\|W_{\psi, \varphi} f_{n}\right\|_{\mathcal{B}_{v}}= & |\psi(0)|\left|f_{n}(\varphi(0))\right|+\sup _{\zeta \in \mathbb{D}} v(\zeta)\left|\left(W_{\psi, \varphi} f_{n}\right)^{\prime}(\zeta)\right| \\
\leq & |\psi(0)|\left|f_{n}(\varphi(0))\right|+\sup _{\zeta \in \mathbb{D}} v(\zeta)\left|\psi^{\prime}(\zeta)\right|\left|f_{n}(\varphi(\zeta))\right| \\
& +\sup _{\zeta \in \mathbb{D}} v(\zeta)\left|\psi(\zeta) \varphi^{\prime}(\zeta)\right|\left|f_{n}^{\prime}(\varphi(\zeta))\right| \\
\leq & |\psi(0)|\left|f_{n}(\varphi(0))\right|+\sup _{\{\zeta \in \mathbb{D}: \varphi(\zeta) \in K\}} v(\zeta)\left|\psi^{\prime}(\zeta)\right|\left|f_{n}(\varphi(\zeta))\right| \\
& +\sup _{\{\zeta \in \mathbb{D}: \delta<<(\zeta) \mid<1\}} v(\zeta)\left|\psi^{\prime}(\zeta)\right|\left|f_{n}(\varphi(\zeta))\right| \\
& +\sup _{\{\zeta \in \mathbb{D}: \varphi(\zeta) \in K\}} v(\zeta)\left|\psi(\zeta) \varphi^{\prime}(\zeta)\right|\left|f_{n}^{\prime}(\varphi(\zeta))\right| \\
& +\sup _{\{\zeta \in \mathbb{D}: \delta<|\varphi(\zeta)|<1\}} v(\zeta)\left|\psi(\zeta) \varphi^{\prime}(\zeta)\right|\left|f_{n}^{\prime}(\varphi(\zeta))\right| \\
\leq & |\psi(0)|\left|f_{n}(\varphi(0))\right|+\|\psi\| \mathcal{B}_{v} \sup _{z \in K}\left|f_{n}(z)\right|+N \sup _{z \in K}\left|f_{n}^{\prime}(z)\right| \\
& +C \sup _{\{\zeta \in \mathbb{D}: \delta<|\varphi(\zeta)|<1\}} v(\zeta)\left|\psi^{\prime}(\zeta)\right|\left(\int_{D_{\varphi(\zeta)}(r)} \sigma d A\right)^{-1 / p}\left\|f_{n}\right\|_{A^{p}(\sigma)} \\
& +C \sup _{\{\zeta \in \mathbb{D}: \delta<|\varphi(\zeta)|<1\}} \frac{v(\zeta)\left|\psi(\zeta) \varphi^{\prime}(\zeta)\right|}{1-|\varphi(\zeta)|^{2}}\left(\int_{D_{\varphi(\zeta)(r)}} \sigma d A\right)^{-1 / p}\left\|f_{n}\right\|_{A^{p}(\sigma)},
\end{aligned}
$$

where we have used the fact that $\psi \in \mathcal{B}_{\nu}$ and $N=\sup _{\zeta \in \mathbb{D}} v(\zeta)\left|\psi(\zeta) \varphi^{\prime}(\zeta)\right|<\infty$.

Using (22) and (23) along with facts that

$$
\left|f_{n}(\varphi(0))\right|<\varepsilon, \quad \sup _{z \in K}\left|f_{n}(z)\right|<\varepsilon \quad \text { and } \quad \sup _{z \in K}\left|f_{n}^{\prime}(z)\right|<\varepsilon
$$


for some $N_{0} \in \mathbb{N}$ and for all $n \geq N_{0}$, in (24), we have $\left\|W_{\psi, \varphi} f_{n}\right\|_{\mathcal{B}_{\nu}}<C \varepsilon$ for $n \geq N_{0}$. Since $\varepsilon>0$ is arbitrary, we have that $\left\|W_{\psi, \varphi} f_{n}\right\|_{\mathcal{B}_{v}} \rightarrow 0$ as $n \rightarrow \infty$. Hence $W_{\psi, \varphi}: A^{p}(\sigma) \rightarrow \mathcal{B}_{v}$ is compact.

Conversely, suppose that $W_{\psi, \varphi}: A^{p}(\sigma) \rightarrow \mathcal{B}_{v}$ is compact. Let $\left(\zeta_{n}\right)_{n \in \mathbb{N}}$ be a sequence in $\mathbb{D}$ such that $\left|\varphi\left(\zeta_{n}\right)\right| \rightarrow 1$ as $n \rightarrow \infty$. If such a sequence does not exist, then (i) and (ii) are vacuously satisfied. Let $g_{n}(z)=\tau_{\varphi\left(\zeta_{n}\right)}(z) f_{\varphi\left(\zeta_{n}\right)}(z)$, where $f_{\lambda}$ is defined in (1) and $\tau_{\lambda}$ is defined in (10). Then as in Theorem 1, $\left\|\tau_{\varphi\left(\zeta_{n}\right)}\right\|_{A^{p}(\sigma)} \lesssim 1$ and by Lemma $3,\left\|f_{\varphi\left(\zeta_{n}\right)}\right\|_{A^{p}(\sigma)} \lesssim 1$ and $\left(f_{\varphi\left(\zeta_{n}\right)}\right)_{n \in \mathbb{N}}$ converges to zero uniformly on compact subsets of $\mathbb{D}$ as $n \rightarrow \infty$. Thus $\left\|g_{n}\right\|_{A^{p}(\sigma)} \lesssim 1$ and $\left(g_{n}\right)_{n \in \mathbb{N}}$ converges to zero uniformly on compact subsets of $\mathbb{D}$ as $n \rightarrow \infty$. Since $W_{\psi, \varphi}: A^{p}(\sigma) \rightarrow \mathcal{B}_{v}$ is compact, we have that $\left\|W_{\psi, \varphi} g_{n}\right\|_{\mathcal{B}_{v}} \rightarrow 0$ as $n \rightarrow \infty$. On the other hand, from (15) we have

$$
\left\|W_{\psi, \varphi} g_{n}\right\|_{\mathcal{B}_{v}} \geq \frac{v(z)\left|\psi\left(\zeta_{n}\right) \varphi^{\prime}\left(\zeta_{n}\right)\right|\left|\varphi\left(\zeta_{n}\right)\right|}{1-\left|\varphi^{\prime}\left(\zeta_{n}\right)\right|^{2}}\left(\int_{D_{\varphi\left(\zeta_{n}\right)}(r)} \sigma d A\right)^{-1 / p}
$$

Using these two facts we have that

$$
\lim _{\left|\varphi\left(\zeta_{n}\right)\right| \rightarrow 1} \frac{v(z)\left|\psi\left(\zeta_{n}\right) \varphi^{\prime}\left(\zeta_{n}\right)\right|}{1-\left|\varphi^{\prime}\left(\zeta_{n}\right)\right|^{2}}\left(\int_{D_{\varphi\left(\zeta_{n}\right)}(r)} \sigma d A\right)^{-1 / p}=0,
$$

from which (i) follows.

Let $f_{\lambda}$ be defined in (1). Then $\sup _{n \in \mathbb{N}}\left\|f_{\varphi\left(\zeta_{n}\right)}\right\|_{A^{p}(\sigma)} \lesssim 1$ and $f_{\varphi\left(\zeta_{n}\right)}$ converges to zero uniformly on compact subsets of $\mathbb{D}$ as $n \rightarrow \infty$. Since $W_{\psi, \varphi}: A^{p}(\sigma) \rightarrow \mathcal{B}_{\nu}$ is compact, we have that

$$
\lim _{n \rightarrow \infty}\left\|W_{\psi, \varphi} f_{\varphi\left(\zeta_{n}\right)}\right\|_{\mathcal{B}_{v}}=0
$$

From (19), we have

$$
\begin{aligned}
& v\left(\zeta_{n}\right)\left|\psi^{\prime}\left(\zeta_{n}\right)\right|\left(\int_{D_{\varphi\left(\zeta_{n}\right)}(r)} \sigma d A\right)^{-1 / p} \\
& \leq\left\|W_{\psi, \varphi} f_{\varphi\left(\zeta_{n}\right)}\right\|_{A^{p}(\sigma) \rightarrow \mathcal{B}_{\nu}}+C \frac{v\left(\zeta_{n}\right)\left|\psi\left(\zeta_{n}\right) \| \varphi^{\prime}\left(\zeta_{n}\right)\right|}{1-\left|\varphi\left(\zeta_{n}\right)\right|^{2}}\left(\int_{D_{\varphi(\zeta n)}(r)} \sigma d A\right)^{-1 / p},
\end{aligned}
$$

which along with (25) and (26) implies that

$$
\lim _{\left|\varphi\left(\zeta_{n}\right)\right| \rightarrow 1} v\left(\zeta_{n}\right)\left|\psi^{\prime}\left(\zeta_{n}\right)\right|\left(\int_{D_{\varphi\left(\zeta_{n}\right)}(r)} \sigma d A\right)^{-1 / p}=0,
$$

from which (ii) follows.

Next we characterize the boundedness and compactness of $W_{\psi, \varphi}: A^{p}(\sigma) \rightarrow \mathcal{B}_{v, 0}$.

Lemma 6 Let $r \in(0,1)$ be fixed, $p>0, p_{0}>1, \alpha>-1$, v be a standard weight, $\psi \in H(\mathbb{D})$ and $\varphi \in S(\mathbb{D})$. Assume that $p_{0} \geq p, \sigma \in L_{+}^{1}(\mathbb{D})$ is such that $\sigma(z) /\left(1-|z|^{2}\right)^{\alpha}$ belongs to $B_{p_{0}}(\alpha)$. Then

$$
\lim _{|z| \rightarrow 1} v(z)\left|\psi^{\prime}(z)\right|\left(\int_{D_{\varphi(z)}(r)} \sigma d A\right)^{-1 / p}=0
$$


if and only if $\psi \in \mathcal{B}_{v, 0}$ and

$$
\lim _{|\varphi(z)| \rightarrow 1} v(z)\left|\psi^{\prime}(z)\right|\left(\int_{D_{\varphi(z)}(r)} \sigma d A\right)^{-1 / p}=0
$$

Proof Suppose that (27) holds. Then, since $\sigma \in L^{1}(\mathbb{D})$, we have

$$
v(z)\left|\psi^{\prime}(z)\right| \lesssim v(z)\left|\psi^{\prime}(z)\right|\left(\int_{D_{\varphi(z)}(r)} \sigma d A\right)^{-1 / p} \rightarrow 0
$$

as $|z| \rightarrow 1$. Hence $\psi \in \mathcal{B}_{v, 0}$. On the other hand, since $|\varphi(z)| \rightarrow 1$ implies $|z| \rightarrow 1$, automatically holds.

Conversely, suppose that $\psi \in \mathcal{B}_{v, 0}$ and (28) hold. By (28), for every $\varepsilon>0$, there exists $\delta \in(0,1)$ such that

$$
v(z)\left|\psi^{\prime}(z)\right|\left(\int_{D_{\varphi(z)}(r)} \sigma d A\right)^{-1 / p}<\varepsilon,
$$

when $\delta<|\varphi(z)|<1$.

On the other hand, since $\psi \in \mathcal{B}_{v, 0}$, there exists $\gamma \in(0,1)$ such that

$$
\nu(z)\left|\psi^{\prime}(z)\right| \leq \varepsilon\left(1-\delta^{2}\right)^{(\eta+2) p_{0} / p},
$$

whenever $\gamma<|z|<1$.

Thus if $\gamma<|z|<1$ and $\delta<|\varphi(z)|<1$, we have that (29) holds, while if $\gamma<|z|<1$ and $|\varphi(z)| \leq \delta$, then from (3) we have

$$
\begin{aligned}
\nu(z)\left|\psi^{\prime}(z)\right|\left(\int_{D_{\varphi(z)}(r)} \sigma d A\right)^{-1 / p} & =\frac{v(z)\left|\psi^{\prime}(z)\right|}{\left(1-|\varphi(z)|^{2}\right)^{(\eta+2) p_{0} / p}} \frac{\left(1-|\varphi(z)|^{2}\right)^{(\eta+2) p_{0} / p}}{\left(\int_{D_{\varphi(z)}(r)} \sigma d A\right)^{-1 / p}} \\
& \lesssim \frac{v(z)\left|\psi^{\prime}(z)\right|}{\left(1-\delta^{2}\right)^{(\eta+2) p_{0} / p}}<\varepsilon .
\end{aligned}
$$

Combining (29) and (30), we easily obtain that (27) holds.

The following lemma is proved similarly. Hence we omit the proof.

Lemma 7 Let $r \in(0,1)$ be fixed, $p>0, p_{0}>1, \alpha>-1, v$ be a standard weight, $\psi \in H(\mathbb{D})$ and $\varphi \in S(\mathbb{D})$. Assume that $p_{0} \geq p, \sigma \in L_{+}^{1}(\mathbb{D})$ is such that $\sigma(z) /\left(1-|z|^{2}\right)^{\alpha}$ belongs to $B_{p_{0}}(\alpha)$.

Then

$$
\lim _{|z| \rightarrow 1} \frac{v(z)\left|\psi(z) \varphi^{\prime}(z)\right|}{1-|\varphi(z)|^{2}}\left(\int_{D_{\varphi(z)}(r)} \sigma d A\right)^{-1 / p}=0
$$

if and only if

$$
\lim _{|z| \rightarrow 1} v(z)\left|\psi(z) \varphi^{\prime}(z)\right|=0
$$


and

$$
\lim _{|\varphi(z)| \rightarrow 1} \frac{\nu(z)\left|\psi(z) \varphi^{\prime}(z)\right|}{1-|\varphi(z)|^{2}}\left(\int_{D_{\varphi(z)}(r)} \sigma d A\right)^{-1 / p}=0
$$

Theorem 3 Let $r \in(0,1)$ be fixed, $p>0, p_{0}>1, \alpha>-1, v$ be a standard weight, $\psi \in H(\mathbb{D})$ and $\varphi \in S(\mathbb{D})$. Assume that $p_{0} \geq p, \sigma \in L_{+}^{1}(\mathbb{D})$ is radial and such that $\sigma(z) /\left(1-|z|^{2}\right)^{\alpha}$ belongs to $B_{p_{0}}(\alpha)$. Then $W_{\psi, \varphi}: A^{p}(\sigma) \rightarrow \mathcal{B}_{\nu, 0}$ is bounded if and only if $W_{\psi, \varphi}: A^{p}(\sigma) \rightarrow \mathcal{B}_{v}$ is bounded, $\psi \in \mathcal{B}_{v, 0}$ and

$$
\lim _{|z| \rightarrow 1} v(z)\left|\psi(z) \varphi^{\prime}(z)\right|=0
$$

Proof First suppose that $W_{\psi, \varphi}: A^{p}(\sigma) \rightarrow \mathcal{B}_{\nu, 0}$ is bounded. Then it is obvious that $W_{\psi, \varphi}$ : $A^{p}(\sigma) \rightarrow \mathcal{B}_{v}$ is also bounded. By taking $f(z) \equiv 1 \in A^{p}(\sigma)$, we have that $\psi \in \mathcal{B}_{v, 0}$. By taking $f(z)=z \in A^{p}(\sigma)$ and using the fact that $\psi \in \mathcal{B}_{v, 0}$, we have that (31) holds.

Conversely, assume that $W_{\psi, \varphi}: A^{p}(\sigma) \rightarrow \mathcal{B}_{v}$ is bounded, $\psi \in \mathcal{B}_{v, 0}$ and (31) holds. Then, for each polynomial $p$, we have that

$$
v(z)\left|\left(W_{\psi, \varphi} p\right)^{\prime}(z)\right| \leq v(z)\left|\psi^{\prime}(z)\right||p(\varphi(z))|+v(z)\left|\psi(z) \varphi^{\prime}(z)\right|\left|p^{\prime}(\varphi(z))\right|,
$$

from which, along with $\psi \in \mathcal{B}_{v, 0}$ and (31), it follows that $W_{\psi, \varphi} p \in \mathcal{B}_{v, 0}$. Since the set of all polynomials is dense in $A^{p}(\sigma)$, we have that for every $f \in A^{p}(\sigma)$, there is a sequence of polynomials $\left(p_{n}\right)_{n \in \mathbb{N}}$ such that $\left\|f-p_{n}\right\|_{A^{p}(\sigma)} \rightarrow 0$ as $n \rightarrow \infty$. Hence, by the boundedness of $W_{\psi, \varphi}: A^{p}(\sigma) \rightarrow \mathcal{B}_{\nu}$, we have

$$
\left\|W_{\psi, \varphi} f-W_{\psi, \varphi} p_{n}\right\|_{\mathcal{B}_{v}} \leq\left\|W_{\psi, \varphi}\right\|_{A^{p}(\sigma) \rightarrow \mathcal{B}_{\nu}}\left\|f-p_{n}\right\|_{A^{p}(\sigma)} \rightarrow 0
$$

as $n \rightarrow \infty$. Since $\mathcal{B}_{v, 0}$ is a closed subspace of $\mathcal{B}_{v}$, we have that $W_{\psi, \varphi}\left(A^{p}(\sigma)\right) \subseteq \mathcal{B}_{v, 0}$ and so $W_{\psi, \varphi}: A^{p}(\sigma) \rightarrow \mathcal{B}_{v, 0}$ is bounded.

Theorem 4 Let $r \in(0,1)$ be fixed, $p>0, p_{0}>1, \alpha>-1$, v be a standard weight, $\psi \in H(\mathbb{D})$ and $\varphi \in S(\mathbb{D})$. Assume that $p_{0} \geq p, \sigma \in L_{+}^{1}(\mathbb{D})$ is radial and such that $\sigma(z) /\left(1-|z|^{2}\right)^{\alpha}$ belongs to $B_{p_{0}}(\alpha)$ and $W_{\psi, \varphi}: A^{p}(\sigma) \rightarrow \mathcal{B}_{v, 0}$ is bounded. Then $W_{\psi, \varphi}: A^{p}(\sigma) \rightarrow \mathcal{B}_{\nu, 0}$ is compact if and only if the following conditions are satisfied:

(i) $\lim _{|z| \rightarrow 1} v(z)\left|\psi^{\prime}(z)\right|\left(\int_{D_{\varphi(z)}(r)} \sigma d A\right)^{-1 / p}=0$;

(ii) $\lim _{|z| \rightarrow 1} \frac{v(z)\left|\psi(z) \varphi^{\prime}(z)\right|}{1-|\varphi(z)|^{2}}\left(\int_{D_{\varphi(z)}(r)} \sigma d A\right)^{-1 / p}=0$.

Proof First suppose that $W_{\psi, \varphi}: A^{p}(\sigma) \rightarrow \mathcal{B}_{v, 0}$ is compact. By taking $f(z) \equiv 1 \in A^{p}(\sigma)$, we have that $\psi \in \mathcal{B}_{v, 0}$. By taking $f(z)=z \in A^{p}(\sigma)$ and using the fact that $\psi \in \mathcal{B}_{v, 0}$, we have that (31) holds. Thus if $\|\varphi\|_{\infty}<1$, then from (3) and the fact that $\psi \in \mathcal{B}_{v, 0}$ we have

$$
\begin{aligned}
& \lim _{|z| \rightarrow 1} v(z)\left|\psi^{\prime}(z)\right|\left(\int_{D_{\varphi(z)}(r)} \sigma d A\right)^{-1 / p} \\
& \quad=\lim _{|z| \rightarrow 1} \frac{v(z)\left|\psi^{\prime}(z)\right|}{\left(1-|\varphi(z)|^{2}\right)^{(\eta+2) p_{0} / p}} \frac{\left(1-|\varphi(z)|^{2}\right)^{(\eta+2) p_{0} / p}}{\left(\int_{D_{\varphi(z)}(r)} \sigma d A\right)^{1 / p}} \\
& \quad \leq C \lim _{|z| \rightarrow 1} \frac{v(z)\left|\psi^{\prime}(z)\right|}{\left(1-\|\varphi\|_{\infty}^{2}\right)^{(\eta+2) p_{0} / p}}=0 .
\end{aligned}
$$


From (3) and (31) we have

$$
\begin{aligned}
& \lim _{|z| \rightarrow 1} \frac{v(z)\left|\psi(z) \varphi^{\prime}(z)\right|}{1-|\varphi(z)|^{2}}\left(\int_{D_{\varphi(z)}(r)} \sigma d A\right)^{-1 / p} \\
& \quad=\lim _{|z| \rightarrow 1} \frac{v(z)\left|\psi(z) \varphi^{\prime}(z)\right|}{\left(1-|\varphi(z)|^{2}\right)^{(\eta+2) p_{0} / p+1}} \frac{\left(1-|\varphi(z)|^{2}\right)^{(\eta+2) p_{0} / p}}{\left(\int_{D_{\varphi(z)}(r)} \sigma d A\right)^{1 / p}} \\
& \quad \leq C \lim _{|z| \rightarrow 1} \frac{v(z)\left|\psi(z) \varphi^{\prime}(z)\right|}{\left(1-\|\varphi\|_{\infty}^{2}\right)^{(\eta+2) p_{0} / p+1}}=0 .
\end{aligned}
$$

Thus in this case conditions (i) and (ii) follow.

Now assume $\|\varphi\|_{\infty}=1$. Let $\left(z_{n}\right)_{n \in \mathbb{N}} \subset \mathbb{D}$ be a sequence such that $\lim _{n \rightarrow \infty}\left|\varphi\left(z_{n}\right)\right|=1$. By the proof of Theorem 2, we have

$$
\lim _{|\varphi(z)| \rightarrow 1} v(z)\left|\psi^{\prime}(z)\right|\left(\int_{D_{\varphi(z)}(r)} \sigma d A\right)^{-1 / p}=0
$$

and

$$
\lim _{|\varphi(z)| \rightarrow 1} \frac{\nu(z)\left|\psi(z) \varphi^{\prime}(z)\right|}{1-|\varphi(z)|^{2}}\left(\int_{D_{\varphi(z)}(r)} \sigma d A\right)^{-1 / p}=0 .
$$

Using (32) and the fact that $\psi \in \mathcal{B}_{v, 0}$, by Lemma 6, we get (i). Using (33) and the fact that $\lim _{|z| \rightarrow 1} v(z)\left|\psi(z) \varphi^{\prime}(z)\right|=0$, by Lemma 7, we get (ii).

Conversely, by taking the supremum in (5) over all $f \in A^{p}(\sigma)$ such that $\|f\|_{A^{p}(\sigma)} \leq 1$ and then letting $|z| \rightarrow 1$, we obtain that

$$
\lim _{|z| \rightarrow 1} \sup _{\|f\|_{A} \|_{(\sigma)} \leq 1} v(z)\left|\left(W_{\psi, \varphi} f\right)^{\prime}(z)\right|=0
$$

Thus, by Lemma 4 , we obtain that $W_{\psi, \varphi}: A^{p}(\sigma) \rightarrow \mathcal{B}_{v, 0}$ is compact.

Note that if $\sigma$ is also a normal weight, then it is easy to see that for a fixed $r \in(0,1)$, the following relationship holds:

$$
\sigma(|\zeta|) \asymp \sigma(|z|), \quad \zeta \in D_{z}(r)
$$

Thus from (34) we have

$$
\int_{D_{\varphi(z)}(r)} \sigma d A \asymp \sigma(|\varphi(z)|)\left(1-|\varphi(z)|^{2}\right)^{2} .
$$

Using (35) and Theorems 1-4, we obtain the following corollaries.

Corollary 1 Let $p>0$, $v$ and $\sigma$ be normal weights, $\psi \in H(\mathbb{D})$ and $\varphi \in S(\mathbb{D})$. Then $W_{\psi, \varphi}$ : $A^{p}(\sigma) \rightarrow \mathcal{B}_{v}$ is bounded if and only if the following conditions are satisfied:

(i) $\widehat{M}_{1}=\sup _{z \in \mathbb{D}} \frac{v(z)\left|\psi^{\prime}(z)\right|}{\sigma(|\varphi(z)|)^{1 / p}\left(1-|\varphi(z)|^{2}\right)^{2 / p}}<\infty$;

(ii) $\widehat{M}_{2}=\sup _{z \in \mathbb{D}} \frac{v(z)\left|\psi(z) \varphi^{\prime}(z)\right|}{\sigma(|\varphi(z)|)^{1 / p}\left(1-|\varphi(z)|^{2}\right)^{1+2 / p}}<\infty$. 
Moreover, if $W_{\psi, \varphi}: A^{p}(\sigma) \rightarrow \mathcal{B}_{v}$ is bounded, then

$$
\left\|W_{\psi, \varphi}\right\|_{A^{p}(\sigma) \rightarrow \mathcal{B}_{v} / \mathbb{C}} \asymp \widehat{M}_{1}+\widehat{M}_{2} .
$$

Corollary 2 Let $p>0, v$ and $\sigma$ be normal weights, $\psi \in H(\mathbb{D}), \varphi \in S(\mathbb{D})$ and $W_{\psi, \varphi}$ : $A^{p}(\sigma) \rightarrow \mathcal{B}_{v}$ be bounded. Then $W_{\psi, \varphi}: A^{p}(\sigma) \rightarrow \mathcal{B}_{v}$ is compact if and only if the following conditions are satisfied:

(i) $\lim _{|\varphi(z)| \rightarrow 1} \frac{v(z)\left|\psi^{\prime}(z)\right|}{\sigma(|\varphi(z)|)^{1 / p}\left(1-|\varphi(z)|^{2}\right)^{2 / p}}=0$;

(ii) $\lim _{|\varphi(z)| \rightarrow 1} \frac{\nu(z)\left|\psi(z) \varphi^{\prime}(z)\right|}{\sigma(|\varphi(z)|)^{1 / p}\left(1-|\varphi(z)|^{2}\right)^{1+2 / p}}=0$.

Corollary 3 Let $p>0$, v and $\sigma$ be normal weights, $\psi \in H(\mathbb{D})$ and $\varphi \in S(\mathbb{D})$. Then $W_{\psi, \varphi}$ : $A^{p}(\sigma) \rightarrow \mathcal{B}_{v, 0}$ is bounded if and only if $W_{\psi, \varphi}: A^{p}(\sigma) \rightarrow \mathcal{B}_{v}$ is bounded, $\psi \in \mathcal{B}_{v, 0}$ and

$$
\lim _{|z| \rightarrow 1} v(z)\left|\psi(z) \varphi^{\prime}(z)\right|=0
$$

Corollary 4 Let $p>0, v$ and $\sigma$ be normal weights, $\psi \in H(\mathbb{D}), \varphi \in S(\mathbb{D})$ and $W_{\psi, \varphi}$ : $A^{p}(\sigma) \rightarrow \mathcal{B}_{v, 0}$ be bounded. Then $W_{\psi, \varphi}: A^{p}(\sigma) \rightarrow \mathcal{B}_{\nu, 0}$ is compact if and only if the following conditions are satisfied:

(i) $\lim _{|z| \rightarrow 1} \frac{v(z)\left|\psi^{\prime}(z)\right|}{\sigma(|\varphi(z)|)^{1 / p}\left(1-|\varphi(z)|^{2}\right)^{2 / p}}=0$;

(ii) $\lim _{|z| \rightarrow 1} \frac{\nu(z)\left|\psi(z) \varphi^{\prime}(z)\right|}{\sigma(|\varphi(z)|)^{1 / p}\left(1-|\varphi(z)|^{2}\right)^{1+2 / p}}=0$.

\section{Competing interests}

The authors declare that they have no competing interests

\section{Authors' contributions}

Both authors contributed equally to the writing of this paper. They read and approved this version of the manuscript.

\section{Author details}

${ }^{1}$ Mathematical Institute of the Serbian Academy of Sciences, Knez Mihailova 36/III, Beograd, 11000, Serbia. ${ }^{2}$ Operator Theory and Applications Research Group, Department of Mathematics, King Abdulaziz University, P.O. Box 80203, Jeddah, 21589, Saudi Arabia. ${ }^{3}$ School of Mathematics, Shri Mata Vaishno Devi University, Kakryal, Katra, JK 182320, India.

Received: 10 August 2015 Accepted: 7 October 2015 Published online: 21 October 2015

\section{References}

1. Colonna, F: New criteria for boundedness and compactness of weighted composition operators mapping into the Bloch space. Cent. Eur. J. Math. 11(1), 55-73 (2013)

2. Colonna, F, Li, S: Weighted composition operators from the minimal Möbius invariant space into the Bloch space. Mediterr. J. Math. 10(1), 395-409 (2013)

3. Li, S, Stević, S: Weighted composition operators from Bergman-type spaces into Bloch spaces. Proc. Indian Acad. Sci. Math. Sci. 117(3), 371-385 (2007)

4. Li, S, Stević, S: Weighted composition operators from $H^{\infty}$ to the Bloch space on the polydisc. Abstr. Appl. Anal. 2007, Article ID 48478 (2007)

5. Li, S, Stević, S: Weighted composition operators between $H^{\infty}$ and $\alpha$-Bloch spaces in the unit ball. Taiwan. J. Math. 12, 1625-1639 (2008)

6. Li, S, Stević, S: Weighted composition operators from Zygmund spaces into Bloch spaces. Appl. Math. Comput. 206(2), 825-831 (2008)

7. Luo, L, Ueki, S: Weighted composition operators between weighted Bergman and Hardy spaces on the unit ball of $\mathbb{C}^{n}$. J. Math. Anal. Appl. 326, 88-100 (2007)

8. MacCluer, B, Zhao, R: Essential norms of weighted composition operators between Bloch-type spaces. Rocky Mt. J. Math. 33(4), 1437-1458 (2003)

9. Sharma, AK, Ueki, S: Composition operators between weighted Bergman spaces with admissible Békollé weights. Banach J. Math. Anal. 8, 64-88 (2014)

10. Stević, S: Essential norms of weighted composition operators from the $\alpha$-Bloch space to a weighted-type space on the unit ball. Abstr. Appl. Anal. 2008, Article ID 279691 (2008)

11. Stević, S: Norm of weighted composition operators from Bloch space to $H_{\mu}^{\infty}$ on the unit ball. Ars Comb. 88, 125-127 (2008)

12. Stević, S: Norms of some operators from Bergman spaces to weighted and Bloch-type space. Util. Math. 76, 59-64 (2008) 
13. Stević, S: Weighted composition operators from weighted Bergman spaces to weighted-type spaces on the unit ball. Appl. Math. Comput. 212, 499-504 (2009)

14. Stević, S, Ueki, SI: Weighted composition operators from the weighted Bergman space to the weighted Hardy space on the unit ball. Appl. Math. Comput. 215, 3526-3533 (2010)

15. Stević, S, Zhou, Z, Chen, R: Weighted composition operators between Bloch type spaces in the polydisc. Sb. Math. 201(1-2), 289-319 (2010)

16. Ueki, Sl: Order bounded weighted composition operators mapping into the Bergman space. Complex Anal. Oper. Theory 6(3), 549-560 (2012)

17. Ueki, SI, Luo, L: Essential norms of weighted composition operators between weighted Bergman spaces of the ball. Acta Sci. Math. 74, 829-843 (2008)

18. Li, S, Stević, S: Composition followed by differentiation between Bloch type spaces. J. Comput. Anal. Appl. 9(2), 195-205 (2007)

19. Li, S, Stević, S: Generalized composition operators on Zygmund spaces and Bloch type spaces. J. Math. Anal. Appl. 338, 1282-1295 (2008)

20. Sharma, AK: Products of multiplication, composition and differentiation between weighted Bergman-Nevanlinna and Bloch-type spaces. Turk. J. Math. 35, 275-291 (2011)

21. Stević, S: On a new integral-type operator from the Bloch space to Bloch-type spaces on the unit ball. J. Math. Anal. Appl. 354, 426-434 (2009)

22. Stević, S: On an integral-type operator from logarithmic Bloch-type and mixed-norm spaces to Bloch-type spaces. Nonlinear Anal. TMA 71, 6323-6342 (2009)

23. Stević, S: Products of integral-type operators and composition operators from the mixed norm space to Bloch-type spaces. Sib. Math. J. 50(4), 726-736 (2009)

24. Stević, S: On an integral operator between Bloch-type spaces on the unit ball. Bull. Sci. Math. 134, 329-339 (2010)

25. Yang, W, Zhu, X: Generalized weighted composition operators from area Nevanlinna spaces to Bloch-type spaces. Taiwan. J. Math. 16(3), 869-883 (2012)

26. Zhu, X: Generalized weighted composition operators from Bloch-type spaces to weighted Bergman spaces. Indian J. Math. 49(2), 139-149 (2007)

27. Madigan, K, Matheson, A: Compact composition operators on the Bloch space. Trans. Am. Math. Soc. 347(7), 2679-2687 (1995)

28. Békollé, D: Inégalite á poids pour le projecteur de Bergman dans la boule unité de $\mathbb{C}^{n}$. Stud. Math. 71, 305-323 (1981/82)

29. Constantin, O: Carleson embeddings and some classes of operators on weighted Bergman spaces. J. Math. Anal. Appl. 365, 668-682 (2010)

30. Constantin, O: Discretizations of integral operators and atomic decomposition in vector-valued weighted Bergman spaces. Integral Equ. Oper. Theory 59, 523-554 (2007)

31. Schwartz, HJ: Composition operators on $H^{p}$. Thesis, University of Toledo (1969)

\section{Submit your manuscript to a SpringerOpen ${ }^{\circ}$ journal and benefit from:}

- Convenient online submission

Rigorous peer review

- Immediate publication on acceptance

- Open access: articles freely available online

- High visibility within the field

- Retaining the copyright to your article 